\title{
BMJ Open Implementation of a lung cancer multidisciplinary team standardised template for reporting to general practitioners: a mixed-method study
}

\author{
Nicole M Rankin, ${ }^{1}$ Gemma K Collett, ${ }^{1}$ Clare M Brown, ${ }^{2}$ Tim J Shaw, ${ }^{3}$ \\ Kahren M White, ${ }^{4}$ Philip J Beale, ${ }^{5}$ Lyndal J Trevena, ${ }^{6}$ Cleola Anderiesz, ${ }^{7}$ \\ David J Barnes ${ }^{2}$
}

To cite: Rankin NM, Collett GK, Brown CM, et al. Implementation of a lung cancer multidisciplinary team standardised template for reporting to general practitioners: a mixedmethod study. BMJ Open 2017;7:e018629. doi:10.1136/ bmjopen-2017-018629

- Prepublication history for this paper is available online. To view these files, please visit the journal online (http://dx.doi. org/10.1136/bmjopen-2017018629).

Received 12 July 2017 Revised 6 November 2017 Accepted 8 November 2017

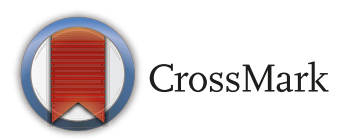

For numbered affiliations see end of article.

Correspondence to Dr Nicole M Rankin; nicole.rankin@ctc.usyd.edu.au

\section{ABSTRACT}

Objectives Few interventions have been designed that provide standardised information to primary care clinicians about the diagnostic and treatment recommendations resulting from cancer multidisciplinary team (MDT) (tumour board) meetings. This study aimed to develop, implement and evaluate a standardised template for lung cancer MDTs to provide clinical information and treatment recommendations to general practitioners (GPs). Specific objectives were to (1) evaluate template feasibility (acceptability, appropriateness and timeliness) with GPs and (2) document processes of preimplementation, implementation and evaluation within the MDT setting.

Design A mixed-method study design using structured interviews with GPs and qualitative documentation of project logs about implementation processes.

Setting Two hospitals in Central Sydney, New South Wales, Australia. Participants: 61 GPs evaluated the template. Two lung cancer MDTs, consisting of 33 clinicians, and eight researchers participated in template development and implementation strategy. Results The MDT-reporting template appears to be a feasible way of providing clinical information to GPS following patient presentation at a lung cancer MDT meeting. Ninety-five per cent of GPs strongly agreed or agreed that the standardised template provided useful and relevant information, that it was received in a timely manner $(90 \%)$ and that the information was easy to interpret and communicate to the patient (84\%). Implementation process data show that the investment made in the preimplementation stage to integrate the template into standard work practices was a critical factor in successful implementation. Conclusions This study demonstrates that it is feasible to provide lung cancer MDT treatment recommendations to GPs through implementation of a standardised template. A simple intervention, such as a standardised template, can help to address quality gaps and ensure that timely information is communicated between tertiary and primary care healthcare providers.
Strengths and limitations of this study

- To the best of our knowledge, this is the first study to report on the implementation of a lung cancer multidisciplinary team standardised template for general practitioners.

- The study highlights the importance of integrating the template into existing work practices to ensure uptake and longer term sustainability.

- Implementation science frameworks are useful in guiding research efforts to integrate standardised templates into routine practice.

- Limitations of conducting the study in two sites are acknowledged, as is the low response rate from general practitioners.

\section{INTRODUCTION}

Multidisciplinary care is considered the best practice in lung cancer management, ${ }^{12}$ providing a comprehensive model within hospital settings that facilitates pathways to diagnosis and treatment. ${ }^{3}$ The benefits of lung cancer multidisciplinary care have been demonstrated across numerous studies. ${ }^{4}$ These include a greater likelihood of treatment receipt or palliative care referral, ${ }^{5}$ guideline-adherent care, improved documentation of disease stage and performance status, ${ }^{5}{ }^{6}$ reduction in time between diagnosis and treatment ${ }^{6}$ and improved patient outcomes and practice patterns. ${ }^{7}$ However, few studies have focused on testing the best practice interventions in lung cancer multidisciplinary team (MDT) settings. ${ }^{89}$

Primary care providers play a significant role in coordinating cancer care ${ }^{10}$ but may not have opportunities to directly participate in hospital-based MDT meetings or processes. ${ }^{11}$ This can limit general practitioners (GPs) access to information about a lung cancer patient's diagnosis and treatment 
decision-making at times of greatest need; these are identified as following presentation at MDT meetings when clinical decisions are being made, ${ }^{12}$ discharge from hospital, changes in a patient's condition and reaching treatment milestones. ${ }^{13}$ In a recent lung cancer qualitative study with GPs, we identified that lack of timely receipt of information from MDTs was a significant source of frustration, and was perceived as impacting on the GP's ability to support the patient and provide timely information. ${ }^{14}$

GPs are the first contact point for many people prior to a lung cancer diagnosis. ${ }^{15-19}$ A study of Australian GPs reported that timely information following presentation of their patient's case at lung cancer MDT meetings is needed, including essential information about diagnosis, and treatment recommendation(s) and intent. ${ }^{13}$ A MDT meeting summary has been suggested as a strategy to facilitate information transfer between GPs and hospital-based clinicians. ${ }^{20}$ Two recent studies have pilot-tested template implementation in a breast cancer MDT setting ${ }^{21}$ and synoptic reporting in colorectal cancer. ${ }^{22}$ There are no previous studies in lung cancer, and thus, it is necessary to first establish intervention feasibility and acceptability prior to full-scale implementation. Frameworks from the implementation science literature provide a basis for documenting the stages of intervention development and testing, which can be summarised as preimplementation, implementation and evaluation. ${ }^{23-25}$

Therefore, the aim of this study was to develop, implement and evaluate a lung MDT meeting standardised template to provide a patient's clinical information and treatment recommendations to the GP following a lung cancer MDT meeting. The specific objectives were to evaluate implementation outcomes of feasibility, timeliness, acceptability and appropriateness and to document the stages of implementation development to enable sustainability and adaptation for other tumour groups.

\section{METHODS}

\section{Study design}

This implementation study used a mixed-method design. We used a structured survey instrument to collect evaluation data from GPs about the template intervention. Qualitative data were collected using a project log to document implementation activities across stages of preimplementation, implementation and evaluation. This study was conducted under the auspices of Cancer Australia's Lung Cancer Demonstration Project, which seeks to develop an evidence-based framework to support national uptake of the 'Principles for best-practice management of lung cancer in Australia'.?

\section{Setting and participants}

The study was conducted within two hospitals located within Sydney Local Health District (SLHD) in metropolitan Sydney, New South Wales. Site A is a large tertiary referral and teaching hospital (over 900 beds) with thoracic surgery, radiation and chemotherapy services provided on site; and, site B is a smaller 750-bed tertiary teaching hospital that provides radiation, chemotherapy and specialised palliative care services. Thoracic oncology MDTs are held weekly at both hospitals, with more than 700 lung cancer cases being presented annually. A 'hub and spoke' model of care was established during the 1980s based on the availability of specialist cardiothoracic surgical procedures at the larger hospital. Two regional hospitals present patient cases on alternate weeks at site A. Participants in the template development and implementation were members of the two lung cancer MDTs $(\mathrm{n}=33)$ and the research team $(\mathrm{n}=8)$.

Participants in the evaluation component were the GPs of patient cases presented at MDT meetings between May 2014 and May 2015. GPs contact details were obtained through Cerner PowerChart electronic medical record (eMR) and entered into an Excel spreadsheet. A project officer contacted every GP office to determine the preferred mode for receiving the template and study documents (via fax, mail or email).

\section{Implementation and evaluation frameworks}

The authors selected the knowledge to action (KTA) cycle framework for our research programme, ${ }^{25}$ in which we have identified significant evidence-practice gaps in lung cancer management, adapted knowledge to the local context through a consensus-driven priority-setting exercise and reported on pathways to diagnosis from patient and GP perspectives via qualitative interviews. ${ }^{4142627}$ The MDT template project was one implementation strategy pilot tested in the 'select, tailor and implement interventions' component of the KTA cycle. Our approach was informed by guidance from the UK Medical Research Council, ${ }^{28}$ health evaluation theory, ${ }^{29}$ Proctor's implementation outcomes framework ${ }^{30}$ and the Expert Recommendations for Implementing Change (ERIC) compilation of implementation strategies. ${ }^{31}$

\section{Preimplementation: Selecting and tailoring the intervention Selection of the intervention}

A self-assessment task was undertaken at project commencement by the authors to identify current gaps in lung cancer management according to a defined set of best practice principles in lung cancer. ${ }^{2} 32$ Our team identified that there was no direct MDT reporting to GPs; this was the responsibility of each patient's coordinating clinician. MDT discussion and consensus about recommended treatment outcomes was typically recorded as free text within the patient's eMR. However, information was inconsistent (that is, no standardisation in items recorded) and was not routinely extracted for reporting to GPs. A generic (non-tumour specific) MDT reporting template had been developed and pilot tested and evaluated with GPs in NSW by one team member (LJT) ${ }^{33}$

\section{Tailoring the generic template}

We commenced tailoring of the generic template to be specific for lung cancer through a consultative process 
with key stakeholders, consisting of 33 MDT members; five local GPs, contacted via the Primary Health Network located in inner western Sydney; three consumers (patients with a previous cancer diagnosis who formed a consumer panel); and three local champions (two MDT Chairs, the Director of Cancer Services, SLHD). All MDT clinicians across the two sites $(n=33)$ gave feedback on the template content and format during a MDT meeting or via email. Standardised items that were routinely reported or discussed during the MDT meeting regarding the patient's history and discussion/consensus about treatment plan were selected. The template underwent a final revision by the authors and was approved by the Director of Cancer Services, and subsequently submitted to eMR team of the Information Management and Technology Division of SLHD for integration into PowerChart eMR.

\section{Intervention content}

As shown in figure 1, the template consists of four sections: preliminary information (15 items), presenting medical officer's information (2 items), clinical items ( 7 items) and summary discussion (3 items).

\section{Implementation processes for template completion}

Integration of the template into eMR systems enabled existing information to be used to prepopulate preliminary items for each patient. Items were checked by a medical registrar (respiratory medicine, medical oncology) during meeting preparation time (about 5 days prior); the remaining items were completed during the meeting. A project officer was responsible for checking the item completeness (GKC). The template content was verified by the nursing case manager for thoracic surgery within 48 hours of the meeting and any missing information was completed (CMB). The MDT Chairs had overall clinical responsibility and answered any queries in the event of missing information.

\section{Measures, recruitment and procedures for evaluation}

Quantitative measure

A structured interview for GPs was developed by the team and reviewed by GPs $(\mathrm{n}=5)$ and the consumer stakeholders described earlier $(n=3)$. The interview asked GPs to evaluate template feasibility according to: (1) appropriateness, (2) timeliness, (3) ease of interpretation and (4) usefulness of treatment recommendations. The interview schedule was pilot tested with the first five participants and responses were reviewed by a second author (NMR) before further interviews were administered. The MDT template and study documents (letter of invitation, information sheet and consent form) were sent to GPs by fax or postal mail with the completed template. No incentive or financial reimbursement was offered for participation and the researchers had no prior relationships with any participating GPs. Consenting GPs were contacted at their workplace by telephone within 2 weeks of the template being sent out. The interview took an average of 10 min to complete.

\section{Lung MDT Form}

Patient Mex:
Age: Sex:
Associated Diagnoses:
VISIT SUMMARY
Royal Prince Alfred Hospital
Missenden Road
CAMPERDOWNNSW 2050

$\begin{array}{ll}\text { Pt Address: } & \text { Pt Phone: } \\ \text { General Practitioner. } & \text { Address: } \\ \text { GP Phone: } & \text { GP Fax: }\end{array}$

Location: Admission/Attendance:

Date:

\section{Presenting Medical Officer: Participantsinvolved in discussion:}

RelevantMedical History:

ECOG Performance Status(score):

\section{Tumour stage: \\ (table will be provided to GPs with report)}

\author{
Diagnostic Tests \\ Chest X-Ray: \\ CT: \\ PET: \\ Histopathology: \\ Discussion: \\ Consensus/Treatment Plan: \\ GP Follow-up:
}

Figure 1 Lung cancer multidisciplinary team (MDT) standardised template. DOB, date of birth; ECOG, Eastern Cooperative Oncology Group; GP, general practitioner; MDT, multidisciplinary team; MRN, medical record number; NSW, New South Wales; PET, positron emission tomography; Pt, patient.

\section{Qualitative measures}

The project officer maintained a weekly project log in Microsoft Excel to document processes in each implementation stage, using two information sources: MDT 
Table 1 Patient demographics, multidisciplinary team (MDT) reporting and general practitioners (GP) responses

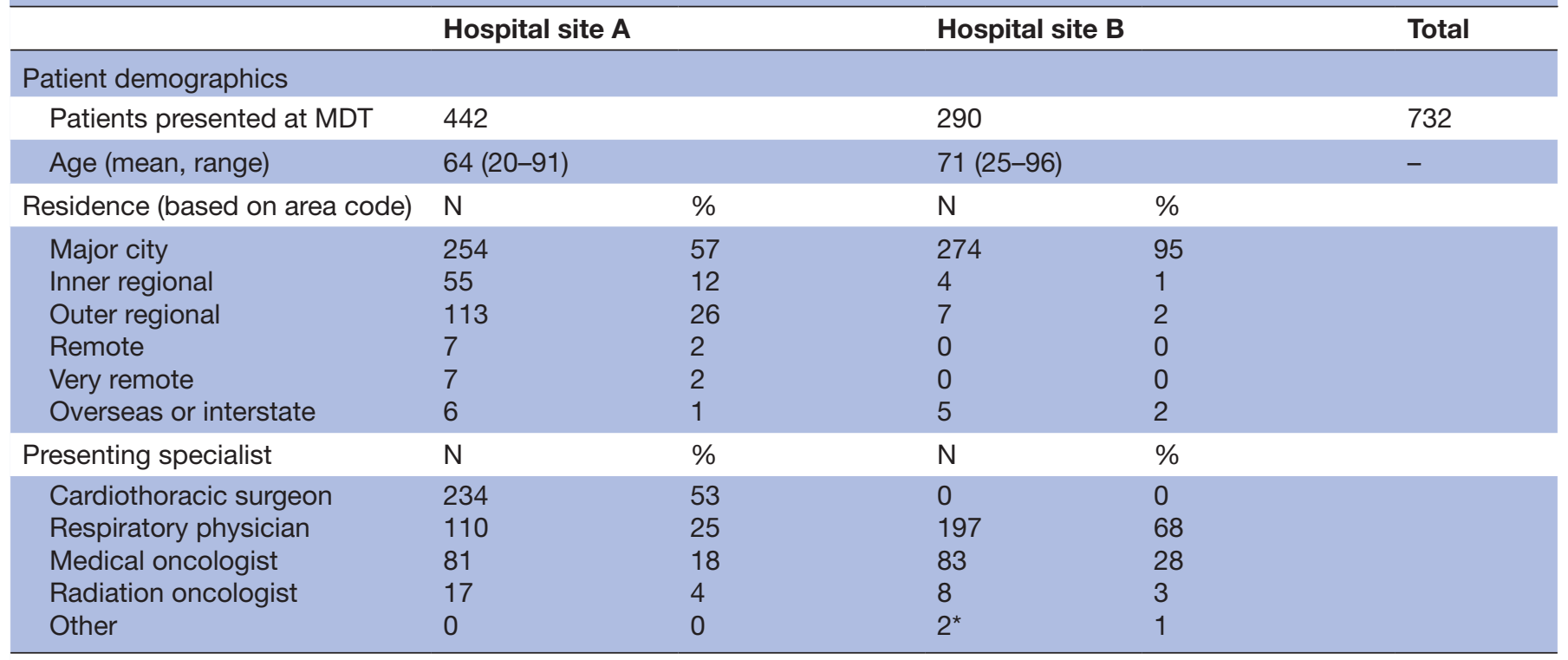

*`Other’ includes one palliative care specialist and one emergency department physician.

meeting minutes and project field notes. Field notes comprised any observations made during MDT meetings about activities that contributed to template completion or implementation processes.

\section{Data collection and analyses \\ Quantitative data}

The project officer recorded interview data in an Excel database during each phone call with GPs. A list of GP contact details was maintained in a separate spreadsheet to deidentified data collected from the surveys. Descriptive statistics were generated in Microsoft Excel and comparative statistics ( $\chi^{2}$ test) in SPSS V.11.

\section{Qualitative data}

Project log data were extracted by arranging content into monthly summaries and analysed for content at the project conclusion by two authors (GKC and NMR). Key activities were grouped into the stages of preimplementation, implementation and evaluation through an iterative process of discussion between authors. The activities were then matched against a compendium of implementation strategies, as described by Powell et al. ${ }^{31}$

\section{RESULTS}

\section{Patient demographics}

Table 1 illustrates the demographic characteristics of the patients presented at lung MDT meetings. Differences in patients' area of residence across the two hospital sites are shown; more patients residing in regional areas were presented at site A, which is consistent with organisation of services. The patient's presenting specialist varied across the sites, with most cases presented by specialist surgeons at site A $(53 \%)$ or by respiratory physicians at site B $(68 \%)$.

\section{GP evaluation}

As shown in table 2, the MDT template was completed for 687 of 732 patients (94\%); of these, 492 patients had a GP listed in their eMR (72\%). All 492 GPs were approached and 61 GPs participated (12\%). Demographic information shows that GPs were evenly split across male and female, were from metropolitan $(57 \%)$ and regional settings $(43 \%)$ and had an average of 21 years' primary care experience (median 20 years; $\mathrm{IQR}=10,30 ; \mathrm{SD}=13.3$ ). Nearly all participants $(98 \%)$ preferred to receive the report by fax.

As shown in table 3, nearly all GPs strongly agreed or agreed that the template information was useful and appropriate, and was received in a timely manner. A large majority of GPs responded that the report would be used for planning the patient's treatment pathway and coordination (agree, 54\%; strongly agree, $32.5 \%$ ). More than $80 \%$ thought that the clinical information provided in the lung MDT report was easy to interpret and communicate to the patient. There were no significant differences in the responses across GPs from metropolitan and regional locations.

Two-thirds of GPs reported that no additional information and/or explanation was required to effectively relay the information to the patient $(66 \%)$. Ten GPs reported wanting additional information, with the remaining $(18 \%)$ being unsure ('neither agree nor disagree'). During the study period, less than 5 of the 492 GPs who received the template chose to contact the thoracic nursing case manager to request further information and/or clarification. Data about whether these GPs contacted a specialist medical clinician about the template were not available.

GPs were asked one open-ended question to give additional feedback about the template. Fourteen participants gave positive feedback that focused on timeliness 
Table 2 Multidisciplinary team (MDT) meeting data and general practitioner (GP) demographics

\begin{tabular}{|c|c|c|c|c|c|}
\hline \multirow[b]{2}{*}{ MDT meeting data } & \multicolumn{2}{|c|}{ Hospital site A } & \multicolumn{2}{|c|}{ Hospital site B } & \multirow[t]{2}{*}{ Total } \\
\hline & $\mathrm{N}$ & $\%$ & $\mathrm{~N}$ & $\%$ & \\
\hline MDT reports generated & 442 & 100 & $245^{\star}$ & 84 & 687 \\
\hline GP survey completed & 52 & 16 & 9 & 5 & 61 \\
\hline \multicolumn{6}{|l|}{ GP demographics } \\
\hline Female & 26 & 50 & 4 & 44 & \\
\hline Male & 26 & 50 & 5 & 56 & \\
\hline Years as GP (mean, range) & \multicolumn{2}{|c|}{$19(1-48)$} & \multicolumn{2}{|c|}{$32(16-52)$} & \\
\hline GP location & $\mathrm{N}$ & $\%$ & $\mathrm{~N}$ & $\%$ & \\
\hline Metropolitan & 26 & 50 & 9 & 100 & \\
\hline
\end{tabular}

There were two reasons for not sending a GP report: (1) no current GP was listed for the patient, or (2) the patient resided overseas.

${ }^{*} n=45$ cases incomplete for MDT reporting.

and content (eg, good timing, quick report, good treatment plan and summary, good to be informed, helpful and relevant). Nineteen participants gave suggestions for improvement about: content $(n=13)$; delivery $(n=4)$ or presentation style $(n=2)$. Ten of the 'content' suggestions were for further information (eg, future tests, appointments, referrals and treatment planning; chemotherapy regimens; psychosocial information). The four 'delivery' suggestions were to instead receive the template from the referring specialist or by phone call. We note that a small group of GPs will prefer receipt of a specialist letter over a standardised template. Saturation of responses was reached after completion of approximately 35 interviews.

\section{Implementation processes}

Documentation of strategies, activities and outcomes in the stages of preimplementation, implementation and evaluation are summarised in table 4 . In preimplementation stage (period of 7 months), we undertook activities that correspond with 10 different strategies to implement new practices, as described in the ERIC compilation. ${ }^{31}$ For example, MDT meetings were observed by the project officer to map out reporting process of meetings at both hospital sites, in order to assess for readiness for change and identify barriers and facilitators. The mapping process documented the data items that were currently being discussed but not formally captured. The MDT Chairs (local champions) highlighted the importance of training for medical registrars who would be responsible for populating the template and capturing consensus discussion about diagnostic and treatment decision-making. Prior to implementation commencement, a clinical leadership forum was held, consisting of a 1 hour video-conference session that engaged participants from site $\mathrm{B}$ and the two regional hospitals sites (which was part of a 'conduct educational meetings' strategy). The purpose was to promote awareness of the project aims to

Table 3 GP responses to evaluation survey items $(n=61)$

$\begin{array}{llccc} & \begin{array}{l}\text { Strongly } \\ \text { disagree N (\%) }\end{array} & \text { Disagree N (\%) } & \begin{array}{l}\text { Neither agree nor } \\ \text { disagree N (\%) }\end{array} & \begin{array}{l}\text { Strongly } \\ \text { Agree N (\%) }\end{array} \\ \text { agree N (\%) }\end{array}$

GP, general practitioner; MDT, multidisciplinary team. 
Table 4 Summary of key activities by project phase captured in project data log

$\begin{array}{lll}\text { Strategies* }^{*} \quad \text { Key activities Outcomes } & \end{array}$

Preimplementation (October 2014-April 2015)

Assess for readiness and identify barriers and facilitators

- Focused literature review: implementation strategies using templates in oncology

- Review examples of hospital reporting templates within organisation; review generic example from primary care

Conduct local needs Conduct process mapping to identify gaps in lung assessment cancer MDT information and communication provision

Build a coalition/conduct educational meetings

- Discuss staff roles and responsibilities for template completion (project officer, specialist nurses, registrars and chairperson)

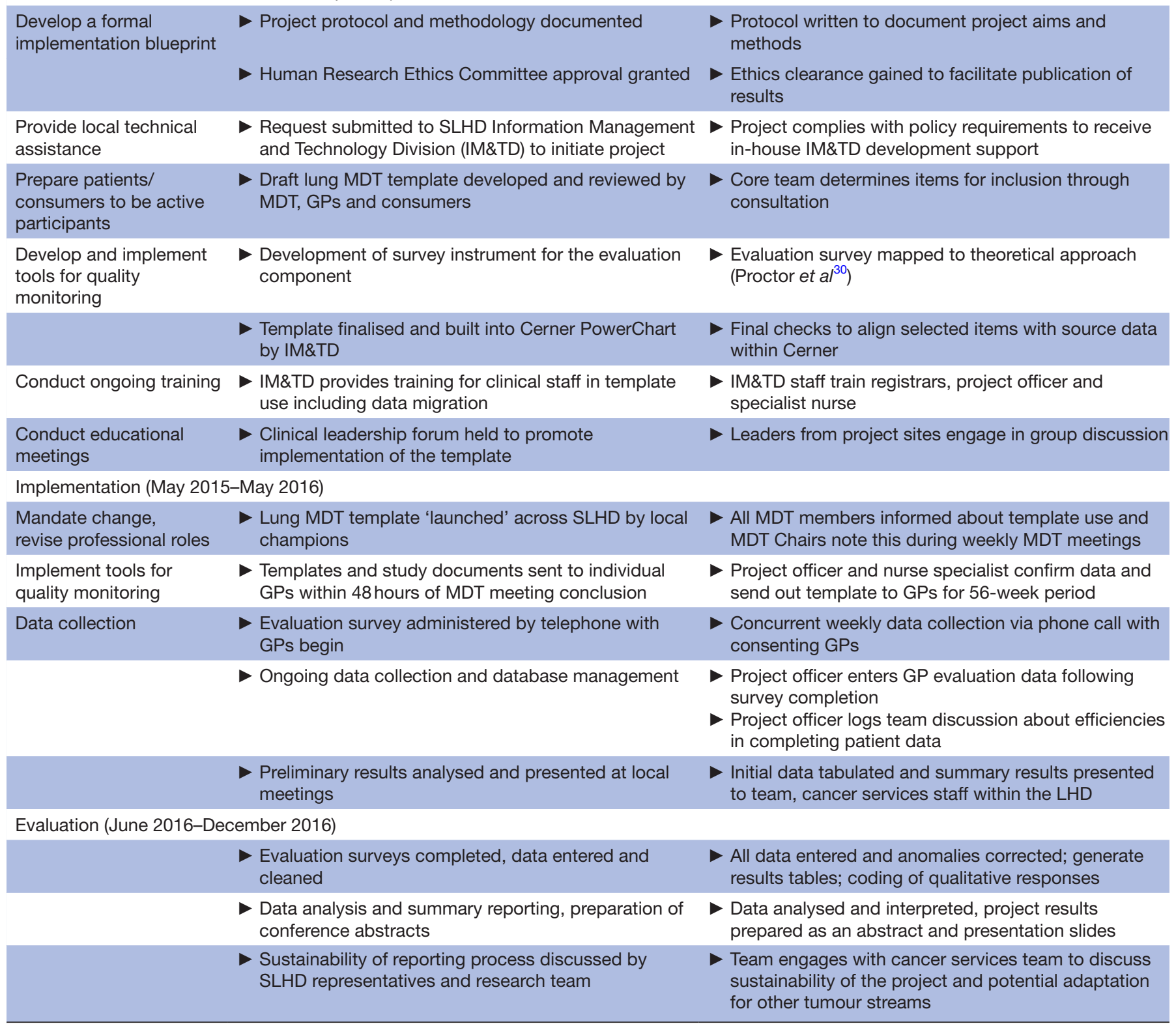

*Strategies as listed in the Expert Recommendations for Implementing Change implementation strategy compilation. ${ }^{31}$ eMR, electronic medical record; GP, general practitioner; LHD, local health district; MDT, multidisciplinary team; SLHD, Sydney Local Health District.

oncology health providers prior to template implementation. The benefits of integration with existing information technology systems were identified by forum participants as crucial to preimplementation, to avoid duplication and ensure compliance with relevant policies and procedures (strategy: provide local technical assistance). 
During implementation (13-month period), the local MDT Chairs mandated change for the routine introduction of the template. The project officer and nurse specialist ensured that each individual template was complete and distributed to the GP within 48 hours $(n=493)$. The project officer collected GP consent forms and conducted evaluation surveys by telephone $(n=61)$. The project officer also logged weekly notes about changes in team efficiency in documenting patients' clinical information, including diagnostic staging and discussion about treatment options. It was noted that the MDT Chairs improved their efficiency to ensure that consensus about diagnosis and treatment recommendations were clearly articulated (or in cases where consensus could not be reached without further investigations) to facilitate clear communication to the registrar who was entering data into each patient's eMR.

The evaluation period ( 7 months) included analysis of GP survey data and initiation of discussion about longer term sustainability of the template within the lung MDT setting and potential adaptation for other tumour groups.

\section{DISCUSSION}

This study aimed to develop, implement and evaluate a standardised template for lung cancer MDT meetings that reports clinical information and treatment recommendations to GPs, following the MDT meeting. The findings show that GPs consider the MDT template to be feasible, appropriate and acceptable; it is delivered in a timely way and contains appropriate information about lung cancer diagnosis and treatment recommendations. It appears that this type of standardised template can be integrated into MDT meeting practice and has the potential to improve information and communication flow across primary and tertiary healthcare sectors. The evaluation data suggest that the template may facilitate discussion between the primary care provider and their patients about diagnosis and treatment options at a time when GPs report most needing this information. ${ }^{13}$

To our knowledge, this is the first study to report on the introduction of a standardised template in lung cancer for GPs. A recent study that introduced a template within a multidisciplinary breast cancer meeting found an increase in recorded adherence rates to national treatment guidelines ${ }^{21}$; however, this study did not use the template as a strategy for communicating with primary care clinicians. Our study demonstrates that a key component preimplementation was template integration into standard MDT workflow practices and electronic systems; this finding is consistent with the breast cancer study, which showed a period of approximately 5 months before the template was fully integrated into workflow practices. ${ }^{21}$ Our findings support the need to introduce electronic solutions to improve access to clinical information by multidisciplinary health professionals..$^{20}$ Ideally, future implementation would include electronic transmission of the template direct to GPs. This is not currently feasible in Australia, which lacks an integrated system of sharing data across all healthcare sectors. ${ }^{34}$

The study's strengths include that we used 10 different preimplementation strategies that engaged key stakeholders (health professionals, information technology staff and consumers), including the conduct of local needs assessment, educational meetings and ongoing training for staff. The standardised template was integrated into existing eMR systems, thus avoiding duplication of resources and facilitated routine collection of data items and reporting to GPs as part of standard component of the best practice. These changes may have encouraged clinical staff to document treatment recommendations into the patient's eMR more thoroughly. Future activities could include an audit and feedback process to report any improvements in practices to the MDTs. We postulate that these steps will contribute to longer term sustainability following the completion of the research project. The project engaged a collaborative team of researchers working in implementation science and oncology and thoracic healthcare professionals and reflects the advantages of interdisciplinary collaboration.

Limitations include that the study was conducted in two hospitals within one health district. However, during the 13-month implementation stage, more than 700 lung cancer cases were presented at MDT meetings. Testing the template in a relatively contained setting helped to show that successful implementation can be achieved across two busy hospital settings. The GP consent rate to participate in evaluation activities was low, and we have previously encountered challenges in engaging GPs in research activities. ${ }^{14}$ We did not have access to data about the non-responding GPs and are therefore unable to comment on whether there were any differences across the groups, or whether this affected the validity of our findings. However, we appear to have reached a broadly representative sample of GPs and heterogeneous spread of responses to evaluation items. We noted qualitative feedback from four GPs that they prefer a specialist letter in preference to a standardised template; the MDT members did not perceive that the template would replace the letter, but instead is delivered as a means of timely communication to the GP. Template information about treatment recommendations may become redundant if patient preferences are not in alignment; however, the GP will have a record of the recommendations to include in the patient's medical history. These limitations of the intervention, rather than of the implementation design, should be canvassed with MDTs if they consider adapting the template for local use. Future improvements could include an item to document which clinician will take responsibility for communicating the MDT recommendation to the patient.

This project provides evidence about the feasibility and acceptability of a standardised template for GP reporting for lung cancer MDTs. Improvements to patient care and management needs to be evaluated to include the impact on patient-GP communication about diagnosis and 
recommended treatment(s). The template can be readily adapted for other MDT tumour groups, as can the processes for implementation and evaluation. Within SLHD, four other tumour groups (two hepatocellular, one haematology and one upper gastrointestinal) have commenced work to modify the template to suit their needs and replicate implementation processes. The template could also support collection of data for reporting against key performance indicators to government cancer control agencies.

\section{CONCLUSIONS}

This study demonstrates that it is feasible to implement a template for GPs reporting clinical information and treatment recommendations for patients with lung cancer discussed at a MDT meeting. Communication between hospital-based MDTs and primary care clinicians can be addressed through implementation of a template intervention. Evaluation of the intervention showed that it was delivered in a manner that was timely, acceptable and appropriate. It is anticipated that it will benefit GPs in communicating the outcomes of MDT treatment recommendations to their patients. The template can be translated to other MDTs for different tumour groups.

\section{Author affiliations}

${ }^{1}$ Sydney Medical School, Sydney Catalyst Translational Cancer Research Centre,

University of Sydney, Camperdown, New South Wales, Australia

${ }^{2}$ Cancer Services, Royal Prince Alfred Hospital, Sydney Local Health District,

Camperdown, New South Wales, Australia

${ }^{3}$ Research in Implementation Science and eHealth (RISe), Faculty of Health Sciences, University of Sydney, Camperdown, New South Wales, Australia

${ }^{4}$ Cancer Institute NSW, Eveleigh, New South Wales, Australia

${ }^{5}$ Cancer Services, Concord Repatriation and General Hospital Cancer Centre, Sydney Local Health District, Concord, New South Wales, Australia

${ }^{6}$ Discipline of General Practice, School of Public Health, University of Sydney, Sydney, New South Wales, Australia

${ }^{7}$ Cancer Australia, Sydney, New South Wales, Australia

Acknowledgements We are very grateful to all project participants, particularly the general practitioners who took part in the evaluation survey and the medical registrars at the participating sites. We acknowledge input of the NSW Collaboration consumer representatives of the Lung Cancer Demonstration Project (LCDP) who willingly shared their experiences and knowledge. We thank the staff of Information Management and Technology Division of Sydney Local Health District for their participation and willingness to support the project. We also thank Barbara Daveson and David Salvestrin from Cancer Australia for supporting the NSW Collaboration of the LCDP.

Contributors This study was proposed by DJB, TJS and NMR. NMR wrote the first draft of the manuscript and contributions were made by DJB, GKC, CMB, TJS, KMW, PJB, LJT and CA. DJB and PJB are clinical leaders at the participating sites. Project implementation was overseen by NMR and TJS. GKC undertook all project management tasks, including ethics and governance approvals, data collection and liaison with the Information Management and Technology Division. GKC and CMB were responsible for sending out templates and liaising with primary care practices with clinical oversight by DJB. Primary care clinical advice for template design was provided by LJT. KMW and CA provided strategic advice about jurisdictional cancer control issues. All authors approved the final draft of the manuscript.

Funding This work was supported by Cancer Australia as part of the NSW Collaboration of the Lung Cancer Demonstration Project (LCDP). The LCDP is an initiative of Cancer Australia, funded by the Australian Government.

Disclaimer The study funder, Cancer Australia, had no role in the study design; the collection, analysis and interpretation of data; or in the decision to submit the article for publication.
Competing interests None declared.

Ethics approval Ethics approval for this project was obtained from the Sydney Local Health District Human Research Ethics Committee (LNRSSA/15/RPAH/236) and site-specific local governance approvals were granted prior to project commencement.

Provenance and peer review Not commissioned; externally peer reviewed.

Data sharing statement № additional data are available.

Open Access This is an Open Access article distributed in accordance with the Creative Commons Attribution Non Commercial (CC BY-NC 4.0) license, which permits others to distribute, remix, adapt, build upon this work non-commercially, and license their derivative works on different terms, provided the original work is properly cited and the use is non-commercial. See: http://creativecommons.org/ licenses/by-nc/4.0/

(c) Article author(s) (or their employer(s) unless otherwise stated in the text of the article) 2017. All rights reserved. No commercial use is permitted unless otherwise expressly granted.

\section{REFERENCES}

1. Conron M, Phuah S, Steinfort D, et al. Analysis of multidisciplinary lung cancer practice. Intern Med J 2007;37:18-25

2. Australian Government Cancer Australia. Principles for best practice management of lung cancer in Australia. 2015 http://canceraustralia. gov.au/sites/default/files/publications/lcbp_principles_for_best practice_management_of_lung_cancer_in_australia_52d60ed4c $2 a 0 d$. pdf.

3. Friedland PL, Bozic B, Dewar J, et al. Impact of multidisciplinary team management in head and neck cancer patients. $\mathrm{Br} J$ Cancer 2011;104:1246-8.

4. Rankin NM, McGregor D, Stone E, et al. Evidence-practice gaps in lung cancer: A scoping review. Eur J Cancer Care 2016.

5. Boxer MM, Vinod SK, Shafiq J, et al. Do multidisciplinary team meetings make a difference in the management of lung cancer? Cancer 2011;117:5112-20.

6. Freeman RK, Van Woerkom JM, Vyverberg A, et al. The effect of a multidisciplinary thoracic malignancy conference on the treatment of patients with lung cancer. Eur J Cardiothorac Surg 2010;38:1-5.

7. Coory M, Gkolia P, Yang IA, et al. Systematic review of multidisciplinary teams in the management of lung cancer. Lung Cancer 2008;60:14-21.

8. Aveling EL, Martin G, Jiménez García S, et al. Reciprocal peer review for quality improvement: an ethnographic case study of the Improving Lung Cancer Outcomes Project. BMJ Qual Saf 2012;21:1034-41.

9. Rummans TA, Clark MM, Sloan JA, et al. Impacting quality of life for patients with advanced cancer with a structured multidisciplinary intervention: a randomized controlled trial. J Clin Oncol 2006;24:635-42.

10. Rubin G, Berendsen A, Crawford SM, et al. The expanding role of primary care in cancer control. Lancet Oncol 2015;16:1231-72.

11. Mitchell GK. The role of general practice in cancer care. Aust Fam Physician 2008;37:698-702.

12. Borras JM, Albreht T, Audisio R, et al.Policy statement on multidisciplinary cancer care. Eur J Cancer 2014;50:475-80.

13. Rowlands S, Callen J, Westbrook J. What information do general practitioners need to care for patients with lung cancer? A survey of general practitioners perceptions. Him J 2010;39:8-16.

14. Rankin NM, York S, Stone E, et al. Pathways to lung cancer diagnosis: a qualitative study of patients and general practitioners about diagnostic and pretreatment intervals. Ann Am Thorac Soc 2017;14:742-53.

15. Largey G, Chakraborty S, Tobias T, et al. Audit of referral pathways in the diagnosis of lung cancer: a pilot study. Aust $J$ Prim Health 2015;21:106-10.

16. Barrett $\mathrm{J}$, Hamilton W. Pathways to the diagnosis of lung cancer in the UK: a cohort study. BMC Fam Pract 2008;9:31.

17. Klabunde CN, Marcus PM, Han PK, et al. Lung cancer screening practices of primary care physicians: results from a national survey. Ann Fam Med 2012;10:102-10.

18. Latimer KM, Mott TF. Lung cancer: diagnosis, treatment principles, and screening. Am Fam Physician 2015;91:250-6.

19. Little AG, Gay EG, Gaspar LE, et al. National survey of non-small cell lung cancer in the United States: epidemiology, pathology and patterns of care. Lung Cancer 2007;57:253-60. 
20. Rowlands S, Callen J. A qualitative analysis of communication between members of a hospital-based multidisciplinary lung cancer team. Eur J Cancer Care 2013;22:20-31.

21. Farrugia DJ, Fischer TD, Delitto D, et al. Improved breast cancer care quality metrics after implementation of a standardized tumor board documentation template. J Oncol Pract 2015;11:421-3.

22. Casati B, Haugland HK, Barstad GM, et al. Implementation and use of electronic synoptic cancer reporting: an explorative case study of six Norwegian pathology laboratories. Implement Sci 2014;9:111.

23. Grol R, Wensing M, Eccles M, eds. Improving patient care: The implementation of change in health care. Nerthlands: Wiley Blackwell, 2013.

24. Brownson R, Colditz G, Proctor E. Dissemination and implementation research in health: translating science into practice. Oxford, 2016.

25. Graham ID, Logan J, Harrison MB, et al. Lost in knowledge translation: time for a map? J Contin Educ Health Prof 2006;26:13-24.

26. McGregor D, Rankin N, Butow P, et al. Closing evidence-practice gaps in lung cancer: Results from multi-methods priority setting in the clinical context. Asia Pac J Clin Oncol 2017;13:28-36.

27. Rankin NM, McGregor D, Butow PN, et al. Adapting the nominal group technique for priority setting of evidence-practice gaps in implementation science. BMC Med Res Methodol 2016;16:110.
28. Craig P, Dieppe P, Macintyre S, et al. Developing and evaluating complex interventions: the new Medical Research Council guidance. BMJ 2008;337:a1655.

29. Øvretveit J. Evaluating health interventions: an introduction to evaluation of health treatments, services, policies and organizational interventions. Buckingham, UK: Open University Press, 2000.

30. Proctor E, Silmere H, Raghavan R, et al. Outcomes for implementation research: conceptual distinctions, measurement challenges, and research agenda. Adm Policy Ment Health 2011;38:65-76.

31. Powell BJ, Waltz TJ, Chinman MJ, et al. A refined compilation of implementation strategies: results from the Expert Recommendations for Implementing Change (ERIC) project. Implement Sci 2015;10:21.

32. Australian Government Cancer Australia. Planning for best practice: self-assessment tool: the Lung Cancer Demonstration Project. Sydney, Australia: Cancer Australia, 2014.

33. Hawkey A, Tattersall MH, Trevena LJ. Defining a role for GPs and patients in multidisciplinary team decisions for cancer care. Innovations in Cancer Treatment and Care Conference. Australia: Cancer Institute NSW, 2013.

34. Austalian Institute of Health and Welfare. Australia's Health: 2014, Australia's health no 14 Cat no AUS 178. Canberra: Austalian Institute of Health and Welfare, 2014. 